\title{
Facial protection in the era of COVID-19: a narrative review
}

Dion Tik Shun Li 1, Lakshman Perera Samaranayake 2*, Yiu Yan Leung 1, Prasanna Neelakantan $3^{*}$

1 Division of Oral and Maxillofacial Surgery, Faculty of Dentistry, The University of Hong Kong, Hong Kong

2 Visiting Professor, College of Dental Medicine, University Sharjah; Professor Emeritus, University of Hong Kong, Hong Kong

3 Discipline of Endodontology, Division of Restorative Dental Sciences, Faculty of Dentistry, The University of Hong Kong, Hong Kong

\section{${ }^{*}$ Corresponding authors:}

Professor Lakshman Samaranayake

M28-125, College of Dental Medicine,

University of Sharjah, 27272, United Arab Emirates

E-mail: Isamaranayake@sharjah.ac.ae; lakshman@hku.hk

Telephone: 971-06 5057317; Fax: 971 -06 5585641

Dr. Prasanna Neelakantan

3B55B, The Prince Philip Dental Hospital,

34, Hospital Road, Hong Kong SAR

E-mail: prasanna@hku.hk

Telephone: +85228590581; Fax: +85225599013.

Running title: COVID-19 and Facial protection

Keywords: coronavirus, COVID-19, facial protection, masks.PAPR

\section{Authors' e-mails:}

Dion Tik Shun Li: diontsli@hku.hk

Lakshman Perera Samaranayake: lakshman@hku.hk

Yiu Yan Leung: mikeyyleung@hku.hk

Prasanna Neelakantan: prasanna@hku.hk 


\begin{abstract}
We live in extraordinary times, where COVID-19 pandemic has brought the whole world to a screeching halt. Tensions and contradictions that surround the pandemic ridden world include the availability, and the lack thereof, various facial protection measures to mitigate the viral spread. Here, we comprehensively explore the different type of facial protection measures, including masks, needed both for the pubic and the health care workers (HCW). We discuss the anatomy, the critical issues of disinfection and reusability of masks, the alternative equipment available for the protection of the facial region from airborne diseases, such as face shields and powered air purifying respirators (PAPR), and the skin-health impact of prolonged wearing of facial protection by HCW. Clearly, facial protection, either in the form of masks or alternates, appears to have mitigated the pandemic as seen from the minimal COVID-19 spread in countries where public mask wearing is strictly enforced. On the contrary, the healthcare systems, that appear to have been unprepared for emergencies of this nature, should be appropriately geared to handle the imbalance of supply and demand of personal protective equipment including face masks. These are two crucial lessons we can learn from this tragic experience.
\end{abstract}

\title{
INTRODUCTION
}

The COVID-19 pandemic presents one of the biggest challenges, as we transition into the third decade of the twenty-first century. Collapses in the healthcare system in some parts of the world, global setback of the economy, and shortage of general and healthcare supplies are just some of the issues that policy makers have to cope with daily. Amidst these challenges lies a critical question that confronts both the healthcare workers and the general public: the use of different types of facial protection. While there is no doubt that wearing different types of facial cover including face masks is an important weapon in the prevention of cross infection in the healthcare setting (Meleney FL, 1926), the efficacy of a majority of these appears to be yet unproven, and matter of conjecture and controversy.

In the current setting of the global shortage of surgical masks, many authorities discourage the use of high efficiency masks in order to reserve them for those at the highest risk of contracting the disease, i.e., healthcare providers in direct contact with infected patients. At the other end of the spectrum is the argument that community-wide mask wearing could play a role in source control, which would likely decrease the rate of transmission. Slowing down the speed of spread would "flatten-the-curve" and alleviate the pressure on the healthcare system, thus the quality of health care delivery would not be compromised (Kenyon, 2020). This is founded on the basis that many carriers of the virus are asymptomatic, and the incubation period or the prodrome can be lengthy, prior to the appearance of any tangible 
symptoms. In the absence of personal protection, these 'silent carriers' as well as 'super spreaders` may spread the virus through their respiratory droplets unawares. Moreover, there is a clear lack of consensus on the level of protection required for different specialities of medical and dental professionals who are in contact with potential disease carriers.

Here, we provide a comprehensive review on the different type of facial covers, including masks, available both for the pubic and the health professionals. Notably, we discuss the anatomy, and the critical issues of disinfection and reusability of masks, the alternative equipment available for the protection of the facial region from airborne diseases, such as face shields and powered air purifying respirators (PAPR). The review concludes with the skinhealth impact of prolonged wearing of facial protection, by health care workers.

\section{CLASSIFICATION OF MASKS}

Understanding the different types of masks can initially be intimidating due to their diversity and confusing terminology. This is complicated by the regional differences in regulations and standards. Nevertheless, most authorities divide masks into three tiers: respirator masks, surgical masks, and single-use face masks.

\section{Respirator masks (N95 and FFP2 variants)}

The first tier in the classification of masks comprises the respirator masks. They have the highest filtering capacity of the three tiers. Respirator masks are designed to filter over $90 \%$ of virus-sized pollutant particles in the air, and are tightly fitted onto the face of the wearer. They require fit testing to ensure proper adaptation to the face.

In the U.S., respirator masks, so called N95 masks are certified by the National Institute for Occupational Safety and Health (NIOSH) and are approved for surgical use by the Food and Drug Administration (FDA) (U.S. National Institute for Occupational Safety and Health, 2018). For example, N95 masks have the capability to filter over $95 \%$ of particles of 0.3 microns. The counter part of the North American N95 masks in Europe are FFP2 masks, where they have a similar filtering capacity. The certification standard used for FFP2 masks in Europe is the EN 149:2001 standard.

Currently, The U.S. Centres for Disease Control and Prevention (CDC) does not recommend the use of respirator masks for non-healthcare providers, as these are critical supplies that should be reserved for those at the highest risk of infection (U.S. Food and Drug Administration, 2020). Respirator masks are designed for single use only, and ideally should be changed 
after every patient encounter. Because of the tight fit and high filtering capacity, the wearer of respirator masks might experience shortness of breath and discomfort after prolonged use.

\section{Surgical masks}

The next tier in the classification of facial masks is the surgical mask. These are loosely fitted masks that do not require fit testing, and are routinely used in the healthcare setting when the procedures do not generate a significant amount of aerosol, and when the risk of acquisition of airborne transmissible diseases is low to moderate. Surgical masks are commonly referred as "face masks". However, not all face masks qualify as surgical masks due to the regulatory guidelines in the U.S., to be considered as surgical masks they have to be certified by the ASTM International (previously known as American Society for Testing and Materials) standards authority. In Europe, the surgical masks are certified using the European Standards Organisation (EN).

Based on their filtration efficacy, surgical masks are classified into Levels 1, 2 and 3 in the U.S. system. Level 3 surgical masks have the highest filtration efficacy compared to their counterparts, with an ability to filter over $98 \%$ of particles of 3.0 microns, and a maximum level of fluid resistance. On the other hand, the European system classifies surgical masks into Type I-III, with the filtration capability similar to their US. counterparts. Surgical masks offer protection against droplets from direct spatter, but do not effectively filter small particles. Surgical masks, unlike respirator masks are not tightly fitted around the face, and due to this loose fit they do not protect against leakage from the lateral aspects of the masks. Therefore, the NIOSH does not recommend that surgical masks be used as particulate respirators (U.S. National Institute for Occupational Safety and Health, 2018). Nevertheless, surgical masks might be effective in blocking splashes, sprays, and large respiratory droplets during routine medical or surgical procedures.

Community-wide use of surgical masks remains a controversial topic in the setting of the ongoing COVID-19 pandemic. On the one hand, the World Health Organization issued an interim report on April 6, 2020, stating that the evidence is lacking for the prevention of the acquisition of Covid-19 virus in healthy persons (World Health Organization, 2020). However, it is now generally accepted that universal mask wearing might be beneficial with regards to source control, as they are likely help prevent the direct projectile of virus-containing respiratory droplets and aerosols from infected individuals. In a recent study using model simulations, it was suggested that the broad adoption of masks by the general public could be effective in reducing the community transmission of COVID-19, thus lessening the burden on 
the healthcare system (Eikenberry et al., 2020). The authors concluded that the effect would potentially be greatest when the compliance of mask wearing is high, and when it is combined with other measures such as social distancing.

In another study by Cheng et al., the authors compared the incidence of COVID-19 in Hong Kong Special Administrative Region (HKSAR) where the compliance of face mask usage by the general public was $96.6 \%$, to that of other "non-mask-wearing" countries with similar population density healthcare systems and social distancing measures. It was found that the incidence of COVID-19 within the first 100 days was significantly lower in HKSAR than that of non-mask-wearing regions. The authors concluded that universal mask-wearing might help reduce COVID-19 burden by containing the emission of infected saliva and respiratory droplets from the mildly infected individuals, or those who are asymptomatic (V. C. Cheng et al., 2020).

\section{Single-use face masks}

Single-use face masks do not meet the requirements of surgical masks. The construction of single-use face masks varies, but are typically thin and might consist of only a single layer. Single-use face masks are not normally used in the healthcare setting, if the supply of surgical masks is not a concern. Although single-use face masks generally cannot filter very small particles, they might still be able to block the emission of large droplets and saliva fairly well. Moreover, when the supply of surgical masks and respirator masks are limited even for healthcare workers $(\mathrm{HCW})$, single-use face masks might be a realistic alternative to be used in the community setting.

\section{Cloth masks}

Due to the acute shortage of surgical masks and N95 respirators, some jurisdictions discourage their use by the general public and reserve them for those at the highest risk for viral exposure, such as HCW who are in close contact with infected patients. A recent recommendation by the CDC issued in April 2020 encouraged the use of cloth face coverings in public settings to slow the spread of COVID-19 (U.S. Centers for Disease Control and Prevention, 2020). This is a seemingly sensible solution when proper masks are in critical supply. While cloth masks do not protect against aerosols, they might still play a role in minimizing the spread of the virus, especially the other recommendations are practiced, such as staying home, reducing unnecessary travel, and social distancing. Cloth masks are increasingly being offered at various online shops, and many do-it-yourself versions have been suggested. A comparison of the various masks is shown in Table 1. 


\section{ANATOMY OF MASKS}

While the construction of unregulated single-use face masks is variable, the anatomy of most surgical masks certified by either the ASTM or EN standards are similar (Figures 1 and 2). Surgical masks are commonly made of three layers, with a filter layer placed between two layers of non-woven fabric (ThomasNet, 2020). The outer layer is usually coloured and is a water-resistant layer, while the inner layer is an absorbent layer and is in contact with the skin of the wearer. The middle filter layer is most commonly made of polypropylene, made through a melt-blown technology (ThomasNet, 2020). Surgical masks are usually pleated to allow adjustment of fit around the face of the wearer. At the top part of the surgical mask, there is an adjustable nose clip for the wearer to adjust the shape around the nasal bridge. This reduces the gap between the mask and the face and thus prevents excessive leakage from the margins of the mask. The most common means for the surgical mask to be attached to the face of the wearer are through either ear loops on either sides of the mask, or via head ties for the wearer to tie them around the head at the level above the ears and around the neck. While surgical masks with ear loops are more convenient to wear and remove, those with head ties are adjustable and might allow a tighter fit around the face of the wearer. This could mean that the amount of leakage around the margins of the mask is less in surgical masks with head ties than those with ear loops.

Respirator masks, such as N95 masks, are usually made of, up to four, multiple layers (ThomasNet, 2020) (Figure 1c) i) comprising a non-woven layer which filters particles of 0.5 microns in diameter, ii) an activated carbon layer which filters chemicals, iii) a cotton layer which filters particles of 0.3 microns in diameter, and iv) a second non-woven layer. They might have an optional valve for regulation of breathing. Similar to surgical masks, N95 masks are commonly made by melt-blown technology using polypropylene (ThomasNet, 2020). Respirator masks are then sterilized after they have been manufactured (ThomasNet, 2020).

\section{DISINFECTION OF MASKS}

The shortage of facemasks during the COVID-19 pandemic has created fear and panic amongst health care workers (HCW) in particular as this essential piece of PPE has been in major short supply, leading to prolonged and repetitive wear of a single mask, and re-use of disinfected masks. Although US CDC has issued guidelines stating that a facemask is considered contaminated when it is worn in managing an infected patient, and the facemask should not be reused (Siegel, Rhinehart, Jackson, Chiarello, \& Health Care Infection Control Practices Advisory, 2007), facemasks shortage, has led to their reuse after decontamination and disinfection in a number of jurisdictions of the world. Researchers have therefore 
investigated the possibilities to disinfect facemasks to ease the shortage problem. The practicality of the disinfection process of a used facemask depends on the following criteria:

i. All pathogens are eliminated,

ii. The structure of the facemask is not damaged,

iii. The function of the facemask including filter capacity is maintained,

iv. No residual disinfectant that could cause health hazard.

Different methods of disinfection have been suggested and tested, which could be broadly categorized into heat (dry and moist), chemical or radiation treatment (Cadnum et al., 2020; Lore, Heimbuch, Brown, Wander, \& Hinrichs, 2012).

\section{Disinfection by heat}

Li et al. compared the efficacy of moist heat (8-10 minutes of heating and 5 minutes of steam) and dry heat $(100 \circ \mathrm{C}$ for 15 minutes) to disinfect N95 respirators using single stranded RNA virus bacteriophage MS2 and methicillin-resistant Staphylococcus aureus as the test organisms (Li, Cadnum, Redmond, Jones, \& Donskey, 2020). Their study found that moist heat significantly kills more viruses and bacteria than dry heat. The study, however, did not test the facemask performance after treatment. On the other hand, it was demonstrated that the use of dry heat or heat with humidity (both less than $100^{\circ} \mathrm{C}$ ) maintained the filtration efficiency of the fabric (Liao et al., 2020). It is important to note that the afore-mentioned study only used the melt blown fabric component of N-95 FFRs without the outer protective coating, indicating a worst-case scenario. Ma et al. also found that steam could effectively decontaminated the medical masks and N95 respirators using a model with avian coronavirus, and further tested there were no significant difference in blocking efficacy of the tested masks in steamed time of 20,60 or 120 minutes (Ma et al., 2020).

\section{Disinfection by chemical treatment}

Another method of disinfection of masks is by means of chemical treatment. Cheng et al. from Hong Kong used $7.8 \% \mathrm{H}_{2} \mathrm{O}_{2}$ mist spray on $\mathrm{H}_{1} \mathrm{~N} 1$ virus-inoculated $\mathrm{N} 95$ respirators and found no growth of the viruses in both the outer and inner surfaces of the facemasks (Cheng, Wong, Kwan, Hui, \& Yuen, 2020). The latter chemical, however, may pose a health hazard to the wearer. Viscusi et al. tested the facemasks that were soaked with a bleaching solution of hypochlorite for 30 minutes followed by rinsing, and allowed air dry overnight, and found the off-gassing concentration of chlorine was $0.1 \mathrm{ppm}$ when the facemasks were rehydrated (Viscusi, Bergman, Eimer, \& Shaffer, 2009). Again, the residual chlorine could pose health hazard to the HCW. Moreover, A recent study (Liao et al., 2020) demonstrated that liquids such as alcohol, chlorine-based solutions and soaps were detrimental to N-95-like fabric 
respirators. These agents cased degradation of the static charge in the fabric and decreased the filtration efficiency dramatically.

\section{Disinfection by radiation}

Facemask disinfection by radiation with microwave, gamma ray or ultraviolet germicidal irradiation (UVGI) have also been tested. Microwave has melted the N95 respirators in one study (Viscusi et al., 2009), while gamma ray caused significant reduction in the filtering capacity of the masks (de Man et al., 2020). Both techniques were therefore not recommended. Appropriate frequency ultra violet was found to have no effect on the facemasks filter capacity (Viscusi et al., 2009), but a study found residual viruses in two of the six samples when tested by droplet inoculation of H1N1 viruses (Heimbuch et al., 2011).

What is the best method to disinfect masks? This is a question that lacks clear-cut answers. However, considering a balance between decontamination and potential material damage, it appears that non-chemical approaches are preferable (Liao et al., 2020). Current evidence favors the use of heat over the radiation and chemical means. Short-term treatment with steam is more effective than ultraviolet light for decontamination of N95 respirators and is as effective as peracetic acid and hydrogen peroxide vapor (Li et al., 2020). Heat disinfection of masks, therefore, would not leave chemical residues which could be detrimental to the health of the wearer, whilst maintaining an acceptable degree of integrity and filtering capacity (Liao et al., 2020; Ma et al., 2020).

\section{REUSABLE MASKS}

The extremely contagious nature of pandemic viral diseases such as COVID-19 mandate precautions with necessary protective equipment, not only for HCW but also the general public. That said, there is also a worldwide shortage of masks, specifically those such as the N95 filtering facepiece respirators (FFR). This implies resorting to other mask types, especially those with reusable potential. Furthermore, the increasing time of usage results on significant resistance to breathing, owing to the build-up of moisture. These caveats raise the question: Can masks be reused? If so, the acceptable frequency of repetitive disinfection, and the type of masks that withstand such chemical assault are key questions that need to be resolved.

Additionally, there is evidence to show that SARS-CoV and SARS-CoV-2 can survive on plastic surfaces for up to 72 hours (van Doremalen et al., 2020). The exact duration of their survival on FFRs or other conventional masks remains unknown. Nevertheless, the possibility of "self-contamination" through repeated use of masks cannot be overruled. Therefore, in terms of masks reusability, the following critical questions must be answered: Can these 
masks be sterilized? What is the optimal mode of sterilization such that it kills the viruses but does not affect the properties of the mask? If so, how many times can they be sterilized? As discussed in the previous section, although research in this area is nascent, much more work regarding disinfection of masks need to be done in order to answer these questions. The next section explores the masks and face shields that are designed to be reusable.

As early as 2006, the National Academy of Sciences, USA suggested that it may be better to stock reusable respirators than N95 respirators (The National Academy of Sciences, 2006). Such reusable respirators contain face pieces that can be cleaned and reused, while the exact nature of reusability of the filter cartridge remains unknown (Weiss, Weiss, Weiss, \& Weiss, 2007). Recently the Government of Hong Kong SAR distributed reusable face masks (CuMask+) to its residents. This six-layered, copper-infused mask is claimed to prevent the colonization/immobilization of bacteria and viruses. This mask, which satisfies the American Society for Testing and Materials (ASTM) F2100 Level 1 standard for particle filtration efficiency (PFE), bacterial filtration efficiency (BFE) and resistance to penetration by synthetic blood. It has also been claimed that this mask was effective up to 60 washes. However, it remains unclear if the efficacy to preventing the novel SARS-CoV-2 is retained up to 60 washes.

In a recently published proof-of-concept study, the authors proposed in interesting approach towards 3D printing of custom-made face mask, with discrete manufacturing approaches for the reusable and disposable components (Swennen, Pottel, \& Haers, 2020). Although leakage and virologic testing of these masks have not been performed at the time of publication, 3D-printed face masks appear to be an interesting solution to the current short supply of PPE. However, the reusability of these protective devices remains unknown, as yet.

As mentioned previously, wearing of cloth masks might be an alternative solution when proper masks are in short supply. However, cloth masks wearing is still a controversial topic due to concerns about reusability and proper disinfection. A practical approach to decontamination of cloth masks is to use steam under pressure. Such an approach was proposed and is used commonly in Taiwan, where, cloth masks are decontaminated using short cycles (about 20 minutes) of heating under pressure in a steam/rice cooker. As discussed above, moist heat disinfection of cloth masks significantly reduces the level of bacteriophage MS2 and Methicillin Resistant Staphylococcus aureus (Li et al., 2020). However, the effects of moist heat on the SARS-CoV-2 on cloth masks is still unknown. 
Whether all the mask types can achieve a balance between filtration efficiency and material integrity after repeated use and disinfection remains a conjecture, as yet. Considering that we are on the verge of further impending epidemics and pandemics, scientists should be prudent in proactively developing masks that have reusable potential.

\section{FACE SHIELDS}

A face shield, worn as an additional barrier in front of a face mask during medical and surgical procedures, is an adjunctive personal protective equipment (PPE) available to HCWs (Figure 3). The purpose of a face shield, that usually consists of a clear plastic material, is to protect the mucous membrane of the face (eyes, nose and mouth) from direct splashing, spraying and spatter of blood, saliva, other contaminated bodily fluids and materials, and irrigation fluids during patient treatment. Because most face shields do not form a tight seal around the side of the face and chin area, they do not offer protection against aerosols leaking in from the margins of the face shields. Also, face shields might be subject to glare and fogging (Roberge, 2016). In fact, strong evidence is lacking in terms of the effectiveness of face shields against the transmission of viral respiratory diseases (The National Academy of Sciences, 2010). Considering the above reasons, they are considered an adjunct, and should be used with other PPE, such as masks, and head caps.

Despite some of the disadvantages of face shields, many authors recommend the use of face shields, especially during the current pandemic, when PPE is in short supply (Advani, Smith, Lewis, Anderson, \& Sexton, 2020; Garcia Godoy et al., 2020; Perencevich, Diekema, \& Edmond, 2020). Face shields are robust, durable, easy to disinfect, and can be reused indefinitely in theory. Also, they are easy to manufacture, and no specific materials are required other than a clear material which is easy to acquire. Additionally, wearing of face shields does not jeopardize interpersonal communication: lip reading and interpretation of facial expressions are still possible. This is particularly important for those with hearing disabilities.

During aerosol generating procedures, the CDC recommends that the care provider should wear either: i) a mask and eye googles, ii) a mask with attached face shield, or iii) a face shield that fully covers the front and sides of the face (Centers for Disease Control and Prevention, 2019). The American Dental Association (ADA) also recommends wearing of face shields by Dental Health-care Personnel (DHCP) when treating patients (American Dental Association, 2020). Because it is assumed that even asymptomatic patients can transmit disease, the highest level of PPE available should be used (American Dental Association, 2020). This 
includes wearing face shields or goggles in addition to the different types of masks (American Dental Association, 2020).

\section{POWERED AIR-PURIFYING RESPIRATOR (PAPR)}

Aerosol-generating procedures (AGPs) are intrinsic to the routine practice of dentistry. While N95 respirators manage to filter at least $95 \%$ of particles $<5 \mu \mathrm{m}$ in size, fit tests are required to ensure the masks fit properly on the user by measuring air leakage. Occasionally, individuals fail the fit tests and are deemed not suitable for wearing N95 respirators, or in situations like HCWs working long hours and/or when the heavy growth of the facial air (e.g. beards, moustaches) impedes the mask fit and integrity (McMahon, Wada, \& Dufresne, 2008). Additionally, prolonged wearing of N95 respirators are also known to be uncomfortable because of the increased breathing resistance, and heat and moisture build-up (Roberts, 2014). Hence powered air-purifying respirators (PAPR) have been suggested as a solution to alleviate the foregoing issues.

PAPR is a battery-powered blower that provides positive airflow through a filter, cartridge, or canister to a hood or face piece (Figure 4). When compared to most facemasks, PAPR may offers additional protection. One study has shown that a properly used PAPR offered up to an assigned protection factor (APF) of 1000 when compared to APF of 10 for a N95 respirator (Centers for Disease Control and Prevention, 2018). The air is filtered by high-efficiency particulate air (HEPA) filter or P100 filters, which are both effective in filtering $99.97 \%$ of particle size $0.3 \mu \mathrm{m}$ in diameter (Bollinger, 2005). PAPR is considered to be the alternative when an individual fails an N95 fitting test. It is also suggested to be used in high risk environments like managing patients with airborne diseases or high risks aerosol generating procedures (Howard, 2020). PAPR is also more comfortable than wearing N95 masks especially those working for long hours with physical exertions such as nurses and orthopaedic surgeons (Powell, Kim, \& Roberge, 2017).

There are several drawbacks of using PAPR on top of its higher cost compared to other facial protection equipment. There are specific guidelines in donning and doffing a PAPR to avoid contamination, which require extra training and time (The National Academy of Sciences, 2015). Some designs of PAPR, such as those with a loose-fitting hood, inhibit the use of headlight or loupes during dental procedures. The constant, noise generated by the air-purifier is also an irritant to the patient as well as the HCWs especially in a dental clinic setting. The 
clinicians and the supporting staff, therefore, need to assess the risks/benefits carefully when deciding the necessity of using PAPR in the dental clinic.

\section{FACE MASKS/RESPIRATORS AND SKIN HEALTH}

The COVID-19 pandemic has clearly spotlighted the facial skin damage due to the prolonged use of face masks, particularly by the front line HCWs. Anecdotal reports indicate that FFP3 masks result in pressure damage to the skin, especially at the nasal bridge. These pressure ulcers are defined as "localized damage to the skin and underlying soft tissue over a bony prominence or related to a medical or other device, where the injury occurs due to prolonged periods of pressure or pressure with shear." (European Pressure Ulcer Advisory Panel (EPUAP), National Pressure Ulcer Advisory Panel (NPUAP), Pan Pacific Pressure Injury Alliance (PPPIA), 2014 ; Gefen et al., 2020).

Dermatological issues are also possible with custom-made 3D-printed masks, Prolonged application of these masks may result in allergic and decubitus lesions at the nasal bridge. This is likely to be specifically amplified in HCW who work in virology units that are humid and warm. While adjustments of non-invasive ventilation devices are fairly easy to perform and hence prevent such ulcerations, such adjustments on protective respirators are not possible. One suggestion to mitigate this problem is to use protective hydrocolloid dressings over the nasal bridge (Payne, 2020). In addition, the routine use of skin unguents may mitigate such damage, although there are no clear guidelines on the frequency of such usage.

\section{CONCLUSIONS}

Tensions and contradictions that surround the current pandemic ridden world include the availability, and the lack thereof, various facial protection measures to mitigate the viral spread. Here, we comprehensively explore the different type of facial protection measures available to the public and the health care workers. We discuss the anatomy, the critical issues of disinfection and reusability of masks, the alternates available, such as face shields, cloth masks, and powered air purifying respirators (PAPR), and the skin-health impact of prolonged wearing of facial protection. Evidence favor the widespread use of some form of face covering minimizes the community spread of COVID-19. Ideally, surgical masks and N95 respirators must be discarded after a single use, but reusing them after disinfection might be a viable option. In the event, simple moist heat treatment could be equally effective as chemical disinfection. 


\section{Acknowledgments}

We are grateful to Ms Akashya Krishnamoorthy, SASTRA University, India for the art work of Figure 4.

\section{Author Contributions}

LPS conceptualized the study. All four authors contributed equally to writing the original draft, critical editing of the final manuscript, and read and approved its final version.

\section{Conflict of interest}

None declared.

\section{Funding}

This study received no funds or financial support from any profit or not-for-profit organization. 


\section{REFERENCES:}

Advani, S. D., Smith, B. A., Lewis, S. S., Anderson, D. J., \& Sexton, D. J. (2020). Universal masking in hospitals in the COVID-19 era: Is it time to consider shielding? Infect Control Hosp Epidemiol, 1-2. doi:10.1017/ice.2020.179

American Dental Association (2020). Interim Mask and Face Shield Guidelines. https://success.ada.org/ /media/CPS/Files/COVID/ADA Interim Mask and Face Shield G uidelines.pdf

Bollinger NJ. NIOSH respirator selection logic. 2005. DHHS (NIOSH) Publication No. 2005-100.

Cadnum, J. L., Li, D. F., Redmond, S. N., John, A. R., Pearlmutter, B., \& Donskey, C. J. (2020). Effectiveness of Ultraviolet-C Light and a High-Level Disinfection Cabinet for Decontamination of N95 Respirators. Pathog Immun, 5(1), 52-67. doi:10.20411/pai.v5i1.372

Centers for Disease Control and Prevention. A guide to air-purifying respirators. DHHS (NIOSH) publication No. 2018-176. Aug 2018.

Cheng, V. C., Wong, S. C., Chuang, V. W., So, S. Y., Chen, J. H., Sridhar, S., . . Yuen, K. Y. (2020). The role of community-wide wearing of face mask for control of coronavirus disease 2019 (COVID-19) epidemic due to SARS-CoV-2. J Infect. doi:10.1016/j.jinf.2020.04.024

Cheng, V. C. C., Wong, S. C., Kwan, G. S. W., Hui, W. T., \& Yuen, K. Y. (2020). Disinfection of N95 respirators by ionized hydrogen peroxide during pandemic coronavirus disease 2019 (COVID19) due to SARS-CoV-2. J Hosp Infect. doi:10.1016/j.jhin.2020.04.003

de Man, P., van Straten, B., van den Dobbelsteen, J., van der Eijk, A., Horeman, T., \& Koeleman, H. (2020). Sterilization of disposable face masks by means of standardized dry and steam sterilization processes; an alternative in the fight against mask shortages due to COVID-19. J Hosp Infect. doi:10.1016/j.jhin.2020.04.001

Eikenberry, S. E., Mancuso, M., Iboi, E., Phan, T., Eikenberry, K., Kuang, Y., . . Gumel, A. B. (2020). To mask or not to mask: Modeling the potential for face mask use by the general public to curtail the COVID-19 pandemic. Infect Dis Model, 5, 293-308. doi:10.1016/j.idm.2020.04.001

European Pressure Ulcer Advisory Panel (EPUAP), National Pressure Ulcer Advisory Panel (NPUAP), Pan Pacific Pressure Injury Alliance (PPPIA). Prevention and treatment of pressure ulcers: quick reference guide. Haesler E (ed). Cambridge Media: Perth, Australia; 2014

Garcia Godoy, L. R., Jones, A. E., Anderson, T. N., Fisher, C. L., Seeley, K. M. L., Beeson, E. A., . . Sullivan, P. D. (2020). Facial protection for healthcare workers during pandemics: a scoping review. BMJ Glob Health, 5(5). doi:10.1136/bmjgh-2020-002553

Gefen, A., Alves, P., Ciprandi, G., Coyer, F., Milne, C. T., Ousey, K., . . Worsley, P. (2020). Devicerelated pressure ulcers: SECURE prevention. J Wound Care, 29(Sup2a), S1-S52. doi:10.12968/jowc.2020.29.Sup2a.S1

Heimbuch, B. K., Wallace, W. H., Kinney, K., Lumley, A. E., Wu, C. Y., Woo, M. H., \& Wander, J. D. (2011). A pandemic influenza preparedness study: use of energetic methods to decontaminate filtering facepiece respirators contaminated with $\mathrm{H} 1 \mathrm{~N} 1$ aerosols and droplets. Am J Infect Control, 39(1), e1-9. doi:10.1016/j.ajic.2010.07.004

Howard, B. E. (2020). High-Risk Aerosol-Generating Procedures in COVID-19: Respiratory Protective Equipment Considerations. Otolaryngol Head Neck Surg, 194599820927335. doi:10.1177/0194599820927335

Kenyon, C. (2020). Flattening-the-curve associated with reduced COVID-19 case fatality rates- an ecological analysis of 65 countries. J Infect. doi:10.1016/j.jinf.2020.04.007

Li, D. F., Cadnum, J. L., Redmond, S. N., Jones, L. D., \& Donskey, C. J. (2020). It's Not the Heat, It's the Humidity: Effectiveness of a Rice Cooker-Steamer for Decontamination of Cloth and Surgical Face Masks and N95 Respirators. Am J Infect Control. doi:10.1016/j.ajic.2020.04.012

Liao, L., Xiao, W., Zhao, M., Yu, X., Wang, H., Wang, Q., . . Cui, Y. (2020). Can N95 Respirators Be Reused after Disinfection? How Many Times? ACS Nano. doi:10.1021/acsnano.0c03597

Lore, M. B., Heimbuch, B. K., Brown, T. L., Wander, J. D., \& Hinrichs, S. H. (2012). Effectiveness of three decontamination treatments against influenza virus applied to filtering facepiece respirators. Ann Occup Hyg, 56(1), 92-101. doi:10.1093/annhyg/mer054 
Ma, Q. X., Shan, H., Zhang, C. M., Zhang, H. L., Li, G. M., Yang, R. M., \& Chen, J. M. (2020). Decontamination of face masks with steam for mask reuse in fighting the pandemic COVID19: experimental supports. J Med Virol. doi:10.1002/jmv.25921

McMahon, E., Wada, K., \& Dufresne, A. (2008). Implementing fit testing for N95 filtering facepiece respirators: practical information from a large cohort of hospital workers. Am J Infect Control, 36(4), 298-300. doi:10.1016/j.ajic.2007.10.014

Meleney FL, S. F. (1926). Postopertive haemolytic streptococcus wound infections and their relation to haemolytic streptococcus carriers among the operating personnel. . Surg Gynecol Obstet, 43, 338-342.

Payne, A. (2020). Covid-19: skin damage with prolonged wear of FFP3 masks. BMJ, 369, m1743. doi:10.1136/bmj.m1743

Perencevich, E. N., Diekema, D. J., \& Edmond, M. B. (2020). Moving Personal Protective Equipment Into the Community: Face Shields and Containment of COVID-19. JAMA. doi:10.1001/jama.2020.7477

Powell, J. B., Kim, J. H., \& Roberge, R. J. (2017). Powered air-purifying respirator use in healthcare: Effects on thermal sensations and comfort. J Occup Environ Hyg, 14(12), 947-954. doi:10.1080/15459624.2017.1358817

Roberge, R. J. (2016). Face shields for infection control: A review. J Occup Environ Hyg, 13(4), 235242. doi:10.1080/15459624.2015.1095302

Roberts, V. (2014). To PAPR or not to PAPR? Can J Respir Ther, 50(3), 87-90. Retrieved from https://www.ncbi.nlm.nih.gov/pubmed/26078617

Samaranayake, L. (2018). Essential microbiology for dentistry (5th ed ed.). Churchill, Edinburgh.

Siegel, J. D., Rhinehart, E., Jackson, M., Chiarello, L., \& Health Care Infection Control Practices Advisory, C. (2007). 2007 Guideline for Isolation Precautions: Preventing Transmission of Infectious Agents in Health Care Settings. Am J Infect Control, 35(10 Suppl 2), S65-164. doi:10.1016/j.ajic.2007.10.007

Swennen, G. R. J., Pottel, L., \& Haers, P. E. (2020). Custom-made 3D-printed face masks in case of pandemic crisis situations with a lack of commercially available FFP2/3 masks. Int J Oral Maxillofac Surg, 49(5), 673-677. doi:10.1016/j.ijom.2020.03.015

The National Academy of Sciences: Board on Health Sciences Policy, Institute of Medicine. The Use and Effectiveness of Powered Air Purifying Respirators in Health Care: Workshop Summary. Washington (DC): National Academies Press (US); 2015 May 7.

The National Academy of Sciences: Committee on the Development of Reusable Facemasks for Use During an Influenza Pandemic. Reusability of Face-masks During an Influenza Pandemic: Facing the Flu. Washington, DC: The National Academies Press; 2006. http://www.nap.edu/catalog/11637.html

The National Academy of Sciences: Institute of Medicine (IOM): Preventing Transmission of Pandemic Influenza and Other Viral Respiratory Diseases. Personal Protective Equipment for Healthcare Workers: Update 2010. Washington, DC: Nathional Academics Press, 2010. P. 10.

ThomasNet (2020). How Surgical Masks are Made, Tested and Used. https://www.thomasnet.com/articles/other/how-surgical-masks-are-made/

ThomasNet (2020). How to make N95 masks? https://www.thomasnet.com/articles/plant-facilityequipment/how-to-make-n95-masks/

U.S. Centers for Disease Control and Prevention (2019). Isolation Precautions. https://www.cdc.gov/infectioncontrol/guidelines/isolation/index.html

U.S. Centers for Disease Control and Prevention (2020). Recommendation Regarding the Use of Cloth Face Coverings, Especially in Areas of Significant Community-Based Transmission. https://www.cdc.gov/coronavirus/2019-ncov/prevent-getting-sick/cloth-face-cover.html

U.S. Food and Drug Administration (2020). N95 Respirators and Surgical Masks (Face Masks). https://www.fda.gov/medical-devices/personal-protective-equipment-infectioncontrol/n95-respirators-and-surgical-masks-face-masks 
U.S. National Institute for Occupational Safety and Health (2018). Respirator Trusted-Source Information: Ancillary Respirator Information.

https://www.cdc.gov/niosh/npptl/topics/respirators/disp part/respsource3.html

van Doremalen, N., Bushmaker, T., Morris, D. H., Holbrook, M. G., Gamble, A., Williamson, B. N., ... Munster, V. J. (2020). Aerosol and Surface Stability of SARS-CoV-2 as Compared with SARSCoV-1. N Engl J Med, 382(16), 1564-1567. doi:10.1056/NEJMc2004973

Viscusi, D. J., Bergman, M. S., Eimer, B. C., \& Shaffer, R. E. (2009). Evaluation of five decontamination methods for filtering facepiece respirators. Ann Occup Hyg, 53(8), 815-827. doi:10.1093/annhyg/mep070

Weiss, M. M., Weiss, P. D., Weiss, D. E., \& Weiss, J. B. (2007). Disrupting the transmission of influenza a: face masks and ultraviolet light as control measures. Am J Public Health, 97 Suppl 1, S32-37. doi:10.2105/AJPH.2006.096214

World Health Organization (2020, April 6). Advice on the use of masks in the context of COVID-19. Interim guidance. https://www.who.int/publications-detail/advice-on-the-use-of-masks-inthe-community-during-home-care-and-in-healthcare-settings-in-the-context-of-the-novelcoronavirus-(2019-ncov)-outbreak 


\begin{tabular}{|l|l|l|l|l|}
\hline & N95 respirator & Surgical mask & $\begin{array}{l}\text { Cloth } \\
\text { mask }\end{array}$ & PAPR \\
\hline Required in Healthcare settings & & & \\
\hline $\begin{array}{l}\text { Recommended for community } \\
\text { use by the CDC }\end{array}$ & & & \\
\hline Regulated by the NIOSH & & & \\
\hline Regulated by the ASTM & & & \\
\hline Requires fit testing & & & \\
\hline Loose fitting & & & \\
\hline Custom fit (ideally) & & & \\
\hline $\begin{array}{l}\text { Filters aerosols (particles } \\
<10 u M) *\end{array}$ & & & \\
\hline Filters droplets \\
(particles <10um)*
\end{tabular}

Table 1: Advantages and disadvantages of currently used facial protection measures. CDC: U.S. Centres for Disease Control and Prevention; NIOSH: the National Institute for Occupational Safety and Health; ASTM: ASTM International -previously known as American Society for Testing and Materials; PAPR: Powered air purifying respirator; *(Samaranayake, 2018; dataform various sources) 


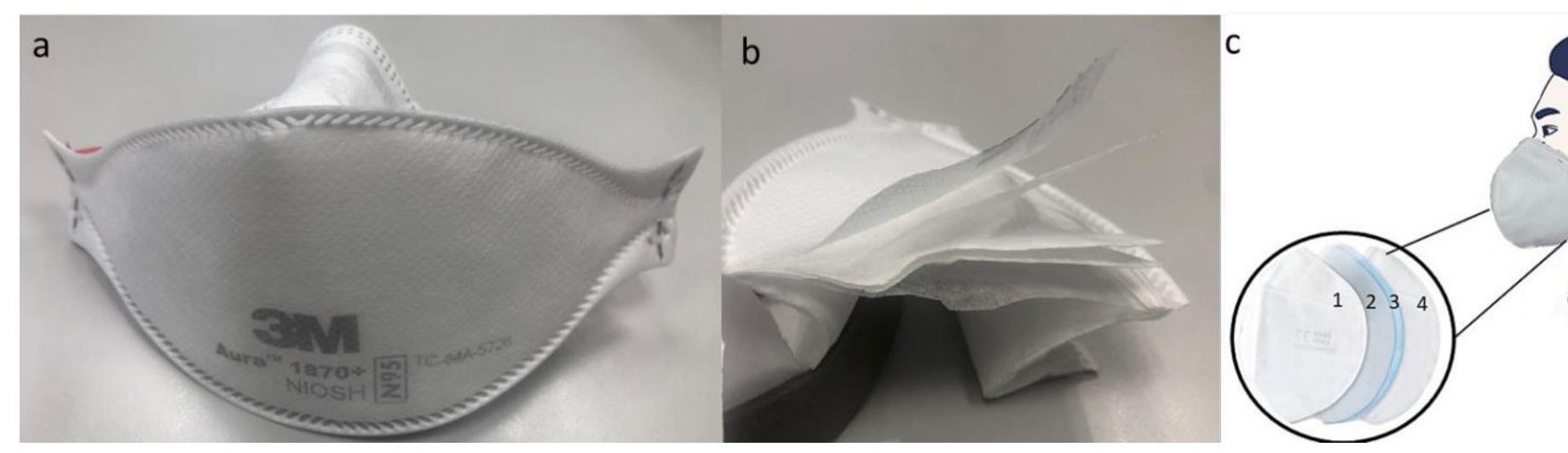

Figure 1. (a) N95 respirator $\left(3 \mathrm{M}^{\mathrm{TM}}\right.$ Aura ${ }^{\mathrm{TM}}$ Health Care Particulate Respirator and Surgical Mask $1870+, \mathrm{N} 95 ; 3 \mathrm{M}^{\mathrm{TM}}$ ), (b) The same respirator cut open to show the multiple-layered construction, (c) Schematic illustration of an N95 respirator which contains: 1) a non-woven layer which filters particles of 0.5 microns in diameter, 2) an activated carbon layer which filters chemicals, 3) a cotton layer which filters particles of 0.3 microns in diameter, and 4) a second non-woven layer.

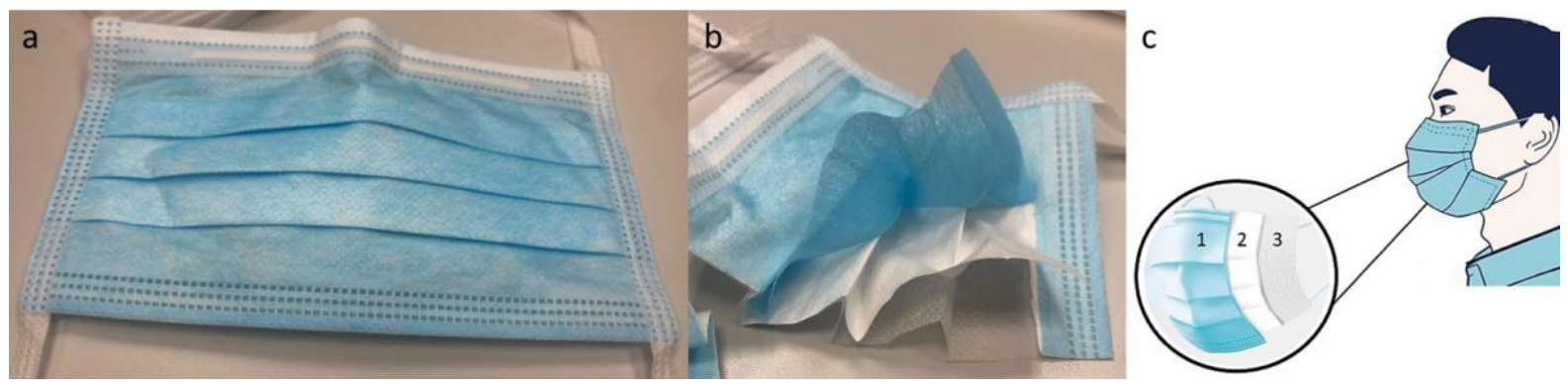

Figure 2. (a) A level 1 (ASTM F2100) tie on surgical mask (Medicom® Tie-On Surgical Mask; A. R. Medicom Inc.), (b) The same surgical mask cut open to show the three-ply construction, (c) Schematic illustration of a surgical mask showing: 1) an outer hydrophobic layer which repels water, blood and bodily fluids, 2) a middle filter layer and 3) an inner hydrophilic layer which absorbs water, sweat and spit. 


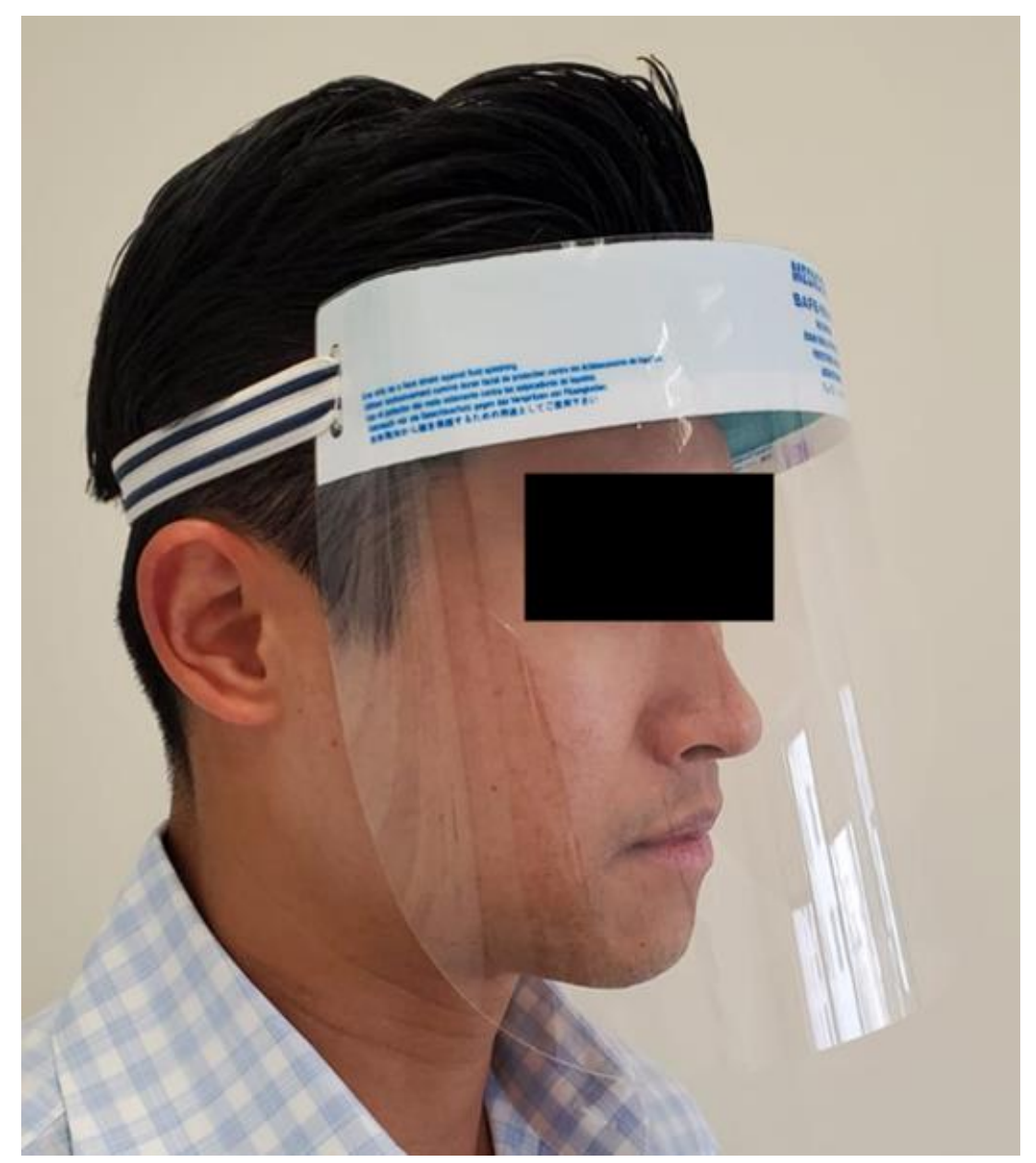

Figure 3. A typical face shield with elastic band around the head (Medicom ${ }^{\circledR}$ Safe+Mask ${ }^{\circledR}$ Face Shield; A. R. Medicom Inc. (Asia) Ltd) 


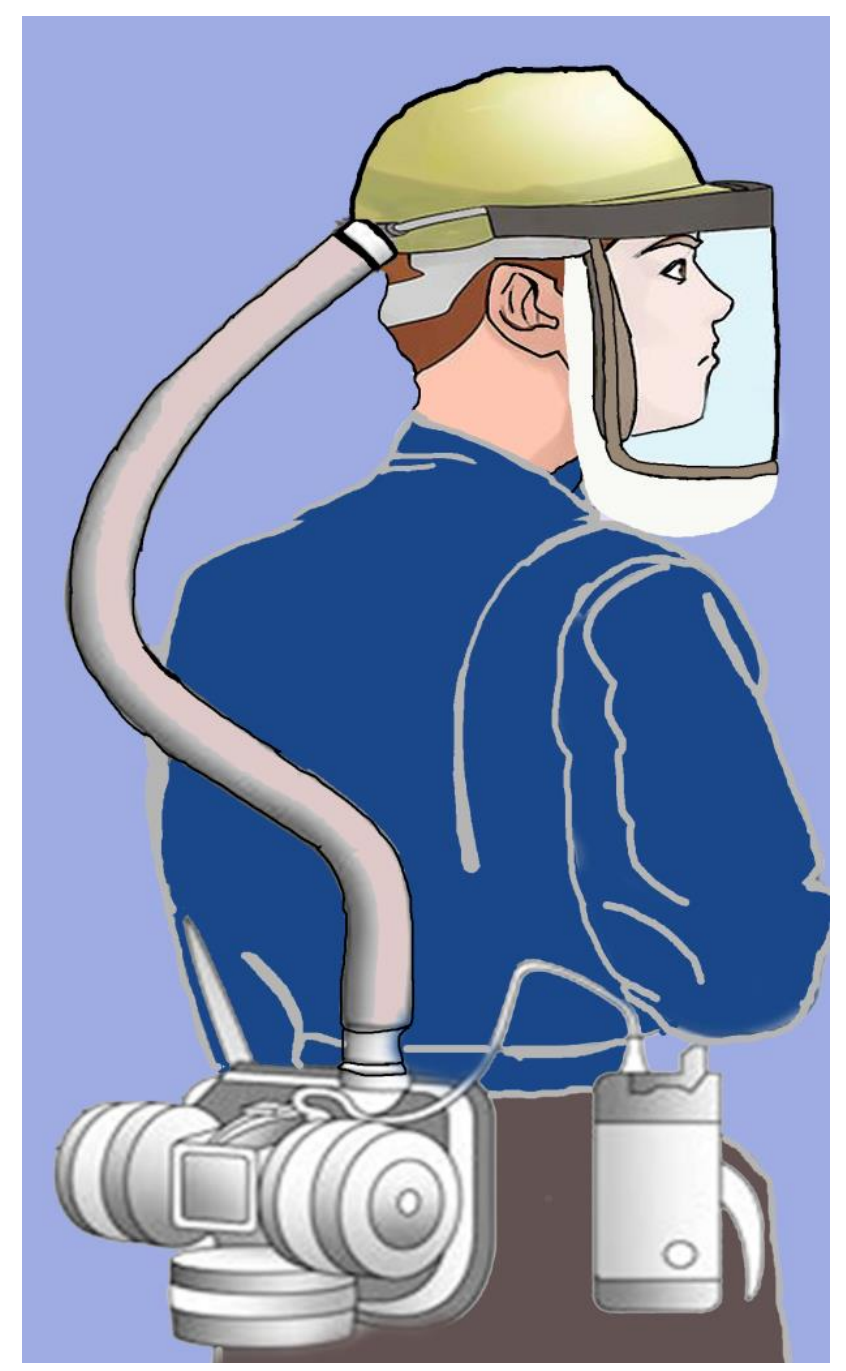

Figure 4. An schematic diagram of a powered air purifying respirator (PAPR) worn by a HCW showing a snugly fitting, air tight, head piece with a transparent plastic lining in front, connected through a (detachable) plastic tube to the power unit. The power unit has a replaceable air filter, and a motor which creates a positive pressure ventilation system with filtered air. 